\title{
Cutaneous Chronic Lymphocytic Leukemia
}

National Cancer Institute

\section{Source}

National Cancer Institute. Cutaneous Chronic Lymphocytic Leukemia. NCI Thesaurus.

Code C45264.

A chronic lymphocytic leukemia that affects the skin. 